\title{
Identification and Outcomes of Hospitalized Medically III Patients Who Are Candidates for Extended Duration Thromboprophylaxis
}

\author{
Craig I. Coleman ${ }^{1,2}$ Gregory Piazza ${ }^{3,4}$ Veronica Ashton ${ }^{5}$ Thomas J. Bunz ${ }^{6}$ Alex C. Spyropoulos ${ }^{7,8}$ \\ 1 Department of Pharmacy Practice, University of Connecticut School \\ Address for correspondence Craig I. Coleman, Pharm D, Department \\ of Pharmacy, Storrs, Connecticut, United States \\ 2 Evidence-Based Practice Center, Hartford Hospital, Hartford, \\ Connecticut, United States \\ ${ }^{3}$ Division of Cardiovascular Medicine, Brigham and Women's \\ of Pharmacy Practice, University of Connecticut, School of Pharmacy, \\ 69 North Eagleville Road, Unit 3092, Storrs, CT 06269, United States \\ (e-mail: craig.coleman@hhchealth.org).
}

Hospital, Boston, Massachusetts, United States

${ }^{4}$ Harvard Medical School, Boston, Massachusetts, United States

${ }^{5}$ Real World Value \& Evidence, Janssen Scientific Affairs, LLC,

Titusville, New Jersey, United States

${ }^{6}$ Division of Pharmacoepidemiology, New England Health Analytics, LLC, Granby, Connecticut, United States

7 Institute for Health Innovations and Outcomes Research, Feinstein Institutes for Medical Research and the Zucker School of Medicine at Hofstra/Northwell, New York, New York, United States

${ }^{8}$ Department of Medicine, Anticoagulation and Clinical Thrombosis Services,

Northwell Health at Lenox Hill Hospital, New York, New York, United States

TH Open 2020;4:e344-e350.

\begin{abstract}
\section{Keywords}

- anticoagulation

- extended duration thromboprophylaxis

- medically ill

- venous thromboembolism

- prevention
\end{abstract}

Background Extended duration thromboprophylaxis (ET) for approximately 30 days can effectively and safely reduce venous thromboembolism (VTE) risk in appropriately selected medically ill patients. We sought to estimate the proportion of hospitalized medically ill patients potentially qualifying for ET and assess their post-discharge clinical and economic outcomes using a large claims database.

Methods Using MarketScan claims from January 2012 to September 2018, we identified medically ill patients hospitalized with a primary diagnosis of heart failure, respiratory insufficiency, ischemic stroke, infection, or inflammatory disease and $\geq 1$-additional risk factor for VTE. Patients $<40$ years old, a length-of-stay $<3$ or $>30$ days, receiving oral anticoagulation prior to index hospitalization or having an indication for full-dose anticoagulation were excluded, as were patients deemed high-risk for bleeding due to active, inhospital treated cancer, gastroduodenal ulcer or bleeding within the prior 3 months, bronchiectasis, pulmonary cavitation or hemorrhage, or dual antiplatelet therapy use.

Results We identified $2,782,988$ patients $\geq 40$ years of age and admitted for a high-risk medical illness. Of these, 724,531 patients $(26.0 \%)$ were identified as ET candidates. Patients' VTE risk appeared highest in the first 30 days post-discharge $(1,532 / 724,531$, $0.2 \%$ ). Adjusted post-index hospitalization costs (2018 US\$) for patients with a VTE within 30 days were higher than those without VTE ( $\Delta=\$ 32,623$ at 30 days, $\Delta=\$ 43,325$ at 90 days, $\Delta=\$ 53,668$ at 365 days; $p<0.001$ for all).

Conclusion Post-discharge VTE in high-risk patients with medical illness is associated with substantially increased costs. received

June 5,2020

accepted after revision

September 22, 2020
DOI https://doi.org/

10.1055/s-0040-1718911. ISSN 2512-9465. (c) 2020 Georg Thieme Verlag KG Stuttgart · New York
License terms

(c) (i) 


\section{Introduction}

Patients with an acute medical illness such as heart failure, respiratory insufficiency, ischemic stroke, infection, or inflammatory disease are highly susceptible to the development of venous thromboembolism (VTE) during their hospital stay and up to 3 months post-discharge, with the highest risk period during the first month. ${ }^{1,2}$ Randomized controlled trial data have demonstrated extended duration thromboprophylaxis (ET) with direct oral anticoagulants for approximately 30 days to prevent VTE which may be associated with a favorable benefit-risk profile when administered to carefully selected patients at high-risk for VTE but lower risk of bleeding. ${ }^{3-6}$ However, there is a relative paucity of studies estimating the proportion of patients that could be considered candidates for ET and assessing their post-discharge incidence of VTE, health care utilization, and costs. In the present study, we sought to estimate the proportion of hospitalized medically ill patients potentially qualifying for ET and assess their post-discharge clinical and economic outcomes using a large claims database.

\section{Methods}

We performed a retrospective analysis using United States IBM MarketScan claims data from January 1, 2012 through September 30, 2018. IBM MarketScan combines two separate databases, a commercial claims and encounters (CCAE) and a Medicare supplemental database, to cover all age groups; and contains claims from approximately 260 contributing employers, approximately 40 health plans and government and public organizations representing approximately 240 million lives. ${ }^{7}$ IBM MarketScan captures enrollment records, demographics, International Classification of Diseases, Ninth- and Tenth-Revision (ICD-9 and ICD-10) diagnosis codes, procedure codes, admission and discharge dates, outpatient medical services data, and prescription dispensing records. All IBM MarketScan data are de-identified and are thus in compliance with the Health Insurance Portability and Accountability Act of 1996 to preserve patient anonymity and confidentiality. This study was determined to not constitute research involving human subjects according to 45 Code of Federal Regulations 46.102(f) and deemed exempt from board oversight.

\section{Patient Identification}

This study defined candidacy for ET as a hospitalization for a medical illness deemed to place a patient at higher risk for developing a VTE but at a lower risk of bleeding according to the criteria utilized in the Multicenter, Randomized, Parallel Group Efficacy and Safety Study for the Prevention of Venous Thromboembolism in Hospitalized Acutely Ill Medical Patients Comparing Rivaroxaban with Enoxaparin (MAGELLAN) subpopulation study. ${ }^{3}$ This study evaluated rivaroxaban versus enoxaparin/placebo for $35 \pm 4$ days to prevent either symptomatic or asymptomatic thrombotic events. The MAGELLAN subpopulation criteria for ET were chosen $a$ priori for this study because they are more easily imple- mented in claims databases as compared with criteria utilized in other ET trials which depend on knowledge of Ddimer levels (which were not available in our MarketScan dataset). ${ }^{3-6}$

To be included in this study, patients had to be hospitalized with a primary discharge diagnosis code for heart failure, respiratory insufficiency, ischemic stroke, infection, or inflammatory (including rheumatic) disease. Patients $<40$ years old, having a length-of-stay (LOS) $<3$ or $>30$ days, dying prior to discharge, having stage 4 or worse chronic kidney disease, receiving oral anticoagulation therapy prior to the index hospitalization or having an indication for fulldose anticoagulation at baseline were excluded. Medically ill patients also had to have at least one additional risk factor for VTE including prolonged immobilization, $\geq 75$ years, morbid obesity, a past medical history of cancer, heart failure or thrombophilia, a personal history of VTE (ICD-10 code of Z86.718 or ICD-9 code of V12.51 only) or an acute infectious disease contributing to the index hospitalization (the latter depicted by the presence of a nonprimary discharge diagnosis code for any infection). ${ }^{3}$ As done in a prior study, ${ }^{8}$ a LOS $\geq 3$ days was considered a proxy for prolonged immobilization. Finally, to identify a cohort of patients at lower risk of bleeding as was done in the MAGELLAN subpopulation analysis, ${ }^{3}$ we excluded patients taking dual antiplatelet therapy or those with active, in-hospital treated cancer, gastroduodenal ulcer or bleeding within the prior 3 months, bronchiectasis, pulmonary cavitation, or hemorrhage.

\section{Outcome Assessment}

Upon the identification of medically ill patients that were candidates for ET, we performed a descriptive analysis to assess patient demographics, baseline comorbidities, index hospitalization characteristics, and post-index hospital discharge outcomes.

The primary outcome of interest was the incidence of 30-day post-discharge VTE. ${ }^{9,10}$ VTE was defined as a hospitalization with a primary discharge diagnosis code for either deep vein thrombosis and/or pulmonary embolism (PE); an emergency department or observation unit encounter with a primary diagnosis code for DVT and/or PE (without a DVT or PE code in the prior 12 months) and accompanied by both a billing code for a VTE diagnostic test (e.g., computed tomography, magnetic resonance imaging, ventilation-perfusion scan, ultrasound) and new initiation of full-dose anticoagulation; or an outpatient visit with a diagnosis code for DVT and/or PE in any position (without a DVT and/or PE code in the prior 12 months) and accompanied by both a billing code for a VTE diagnostic test and new initiation of full-dose anticoagulation. Secondary clinical outcomes included time-topost-discharge VTE, the incidence of rehospitalization for any cause and incidence of a post-index hospitalization major adverse cardiovascular event (defined as a subsequent hospitalization with a primary discharge diagnosis code for myocardial infarction or stroke) at 30 days post-index hospitalization, as well as the incidence of recurrent VTE (second post-discharge VTE 
defined as a hospitalization with a primary diagnosis code for DVT and/or PE) at 365 days post-discharge.

The primary economic outcome for this study was total post-hospital discharge costs, including inpatient, outpatient, and outpatient pharmacy costs (but not including the costs of the index hospitalization). Costs were inflated to 2018 US\$ using the consumer price index for Medical Care available from the Bureau of Labor Statistics. ${ }^{11}$ We compared total costs at 30, 90 and 365 days between patients who developed a post-index hospitalization VTE within 30 days of discharge (early VTE) and those who did not.

Subgroup analysis was performed in which we evaluated the incidence of VTE and total post-hospital discharge health care costs in patients with an additional risk factor for VTE that was present in $>5 \%$ of the ET candidate population (i.e., $\geq 75$ years old, morbid obesity, history of heart failure, history of cancer).

Continuous data were summarized as means \pm standard deviations (SDs). Categorical outcomes were reported as incidences $(n, \%)$; and Kaplan-Meier analysis was performed to assess time-to-first post-index hospitalization VTE. Testing for statistical significance of unadjusted total post-hospital discharge costs between those developing and not developing early VTE was performed using an independent samples $t$-test. Adjusted differences in total post-hospital discharge costs between those developing and not developing early VTE were also estimated using generalized linear regression models including sex, primary reason for the index hospitalization, and 12 comorbidities/risk factors for VTE including history of cancer, history of VTE, history of heart failure, thrombophilia, morbid obesity, acute infectious disease contributing to admission, prolonged immobilization, chronic venous insufficiency, varicosis, recent major surgery or trauma in the prior 6 to 12 weeks, estrogen therapy, and chronic kidney disease.. All data management and statistical analysis were performed using SAS version 9.4 (SAS Institute Inc., Cary, North Carolina, United States) and IBM SPSS Statistics version 25.0 (IBM Corp., Armonk, New York, United States).

This report was written to comply with the Reporting of Studies Conducted using Observational Routinely Collected Health Data (RECORD) statement. ${ }^{12}$

\section{Results}

In total, 10,579,706 unique hospitalized medically ill patients admitted for any reason were included in the MarketScan dataset between January 1, 2012 and September 30, 2018. Of these, 3,236,752 were admitted for heart failure, ischemic stroke, infection, inflammatory/rheumatic, or respiratory disease based upon primary hospital billing codes. Age $<40$ years resulted in the exclusion of 453,764 patients leaving $2,782,988$ patients. After excluding patients with $\operatorname{LOS}<3$ or $>30$ days or dying in-hospital, those already taking or having an indication for oral anticoagulation and those with stage 4 or worse chronic kidney disease, 1,123,347 patients remained eligible. From these, 398,816 were excluded for having at least one of the five major bleeding risk factors identified in the MAGELLAN subpopulation, ${ }^{3}$ leaving 724,531 patients $(26.0 \%$ of $2,782,988)$ as ET candidates. A detailed flow diagram of patient inclusion and exclusion is provided as - Fig. 1.

Characteristics of 724,531 ET candidate patients are described in - Table 1. The most frequent qualifying primary coded cause for the medically ill hospitalization was acute inflammatory or rheumatic disease $(38.2 \%)$, followed by acute respiratory disease (26.4\%), and infection (21.5\%), with acute ischemic stroke and heart failure each making up less than one-tenth of the final study cohort. Other than prolonged immobilization, age $\geq 75$ years, prior history of cancer, heart failure, and morbid obesity were the additional VTE risk factors that had a prevalence $>5 \%$. The index hospitalization LOS averaged $5.4 \pm 3.9$ days and cost a mean of $\$ 33,290 \pm 43,503$ per patient.

The incidence rate of VTE was greatest in the first 30-days post-discharge with $1,532(0.2 \%)$ patients experiencing a VTE (-Fig. 2). Total post-hospital discharge health care costs for patients experiencing an early VTE within 30-days of discharge were higher at $30(\$ 39,558 \pm 73,670$ vs. $\$ 6,626 \pm 22,668), 90$ $(\$ 58,394 \pm 101,396$ vs. $\$ 14,384 \pm 39,964)$, and 365 days $(\$ 88,680 \pm 131,225$ vs. $\$ 33,842 \pm 78,304)$ post-index hospitalization than for patients not experiencing a VTE within 30 days. Unadjusted differences in total costs between those with and without a 30 day post-discharge VTE were $\$ 32,932$ $(p<0.001)$ at 30 days, increased to $\$ 44,010(p<0.001)$ at 90 days, and continued to widen at 365 days $(\$ 54,838$, $p<0.0001$ ) (-Fig. 3). Cost differences between patients with and without a 30 day post-discharge VTE remained significantly different after adjustment using generalized linear modeling $(\$ 32,623$ at 30 days; $\$ 43,325$ at 90 days, and $\$ 53,668$ at 365 days; $p<0.001$ for all).

The incidence of post-discharge VTE and total downstream health care costs in the subset of patients who were $\geq 75$ years old, morbidly obese, or had a history of heart failure or cancer were comparable to the overall ET candidate population (-Table $\mathbf{2}$ ).

The incidence rates of secondary outcomes at 30 days were 50,049 (6.9\%) for rehospitalization for any cause and $1,180(0.2 \%)$ for a major adverse cardiovascular event hospitalization. A second, or recurrent, VTE occurred in 163 of the 4,487 (3.6\%) medically ill patients who developed an initial VTE post-discharge during the 365-day post-discharge period.

\section{Discussion}

The present study found that $26 \%$ of medically ill patients $\geq 40$ years of age hospitalized in routine practice in the United States would qualify for ET with an approved direct oral anticoagulant such as rivaroxaban according to prescribing labeling. ${ }^{13}$ The evaluated medically ill cohort was at highrisk of post-discharge VTE due to patients' primary reason for hospitalization, but at a lower risk of bleeding due to the exclusion of patients with key bleeding risk factors. ${ }^{3}$ In the first 30 -days post-discharge, 1,532 of 724,531 (or $0.2 \%$ ) of evaluated medically ill patients were diagnosed with an 


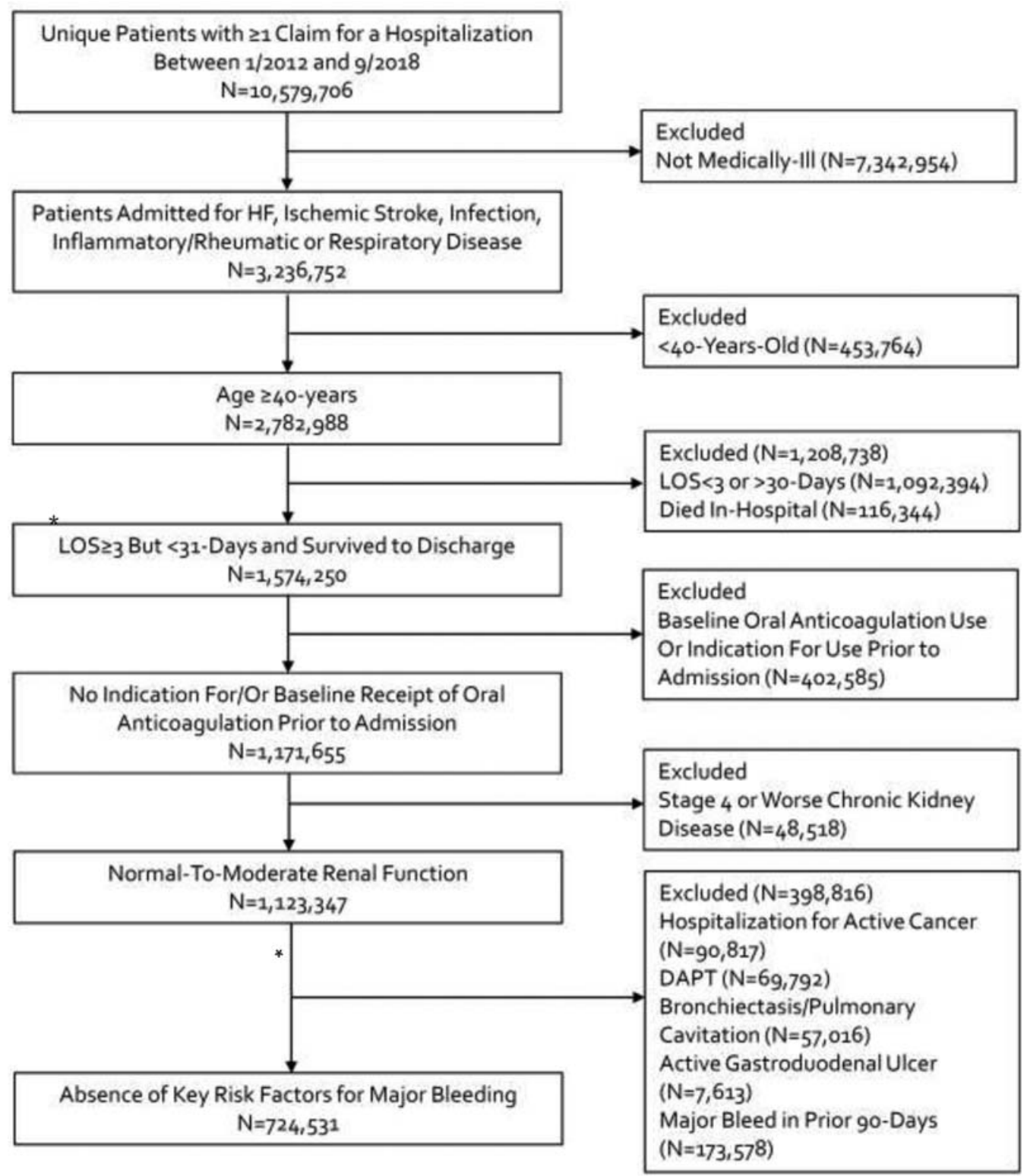

Fig. 1 Inclusion and exclusion of patients. DAPT, dual antiplatelet therapy; HF, heart failure; LOS, length-of-stay; N, number. *Length-of-stay $\geq 3$ days inclusion criteria was a proxy for prolonged immobilization; and therefore, all patients had at least one additional risk factor for the development of venous thromboembolism

acute VTE. Patients experiencing a VTE in the first 30-days had significantly higher total post-hospital discharge health care costs than patients who did not experience a VTE, with substantial cost differences seen early (surpassing $\$ 32,000$ at 30 days, $p<0.001$ ) and approaching $\$ 55,000$ by 1 year $(p<0.0001)$.

The incidence rate of VTE in our study was estimated to be $0.2 \%$ at 30 days and $0.6 \%$ at 365 -days post-discharge. When interpreting our incidence rates several factors need to be considered. Compared with randomized trials ${ }^{3-6}$ and some real-world studies evaluating "hospital-acquired" VTE and its prevention, ${ }^{10,14}$ our study did not count thrombotic events that occurred during the index hospital admission. We opted to not attempt to capture these events as the positive predictive value of identifying acute VTE using nonprimary billing codes (which by default was the case in the present study as we required heart failure, respiratory insufficiency, ischemic stroke, infection, or inflammatory disease to be the primary coded cause) is poor, ranging only between 50 and $75 \%{ }^{9}$ Moreover, randomized trials actively sought to identify asymptomatic VTE which often made up $>75 \%$ of identified cases. ${ }^{3}$ In our real-world dataset, it was unlikely that asymptomatic VTE was commonly assessed or identified. Of note, our reported rate of VTE at 30-days was generally consistent with the incidence of symptomatic VTE reported in the MAGELLAN subpopulation at $35 \pm 4$ days (0.7\%) minus those events occurring during the $10 \pm 4$ days index hospitalization period $(0.5 \%){ }^{3}$ Finally, as IBM MarketScan data ${ }^{7}$ consists largely of commercially insured patients augmented by patients $\geq 65$ years-old with Medicare supplemental insurance, the medically ill cohort evaluated in our study is likely 
Table 1 Preindex hospitalization characteristics of included patients

\begin{tabular}{|l|l|}
\hline Characteristic & $\begin{array}{l}\boldsymbol{N}=724,531 \\
\boldsymbol{n}(\%)\end{array}$ \\
\hline Sex (male) & $405,036(55.9)$ \\
\hline Age (y), mean \pm standard deviation & $64.6 \pm 13.3$ \\
\hline$<65$ y & $421,739(58.2)$ \\
\hline $65-74$ y & $126,368(17.4)$ \\
\hline$\geq 75$ y & $176,424(24.4)$ \\
\hline Reason for hospitalization & $48,099(6.6)$ \\
\hline Heart failure & $53,140(7.3)$ \\
\hline Acute ischemic stroke & $155,794(21.5)$ \\
\hline Acute infectious disease & $276,578(38.2)$ \\
\hline Acute inflammatory or rheumatic disease & $190,920(26.4)$ \\
\hline Acute respiratory disease & $116,403(16.1)$ \\
\hline Additional risk factors for venous thromboembolism \\
\hline History of cancer & $1,432(0.2)$ \\
\hline History of venous thromboembolism & $61,052(8.4)$ \\
\hline History of heart failure & $1,056(0.1)$ \\
\hline Thrombophilia & $176,424(24.4)$ \\
\hline Age $\geq 75$ y & $39,848(5.5)$ \\
\hline Morbid obesity & $32,282(4.5)$ \\
\hline $\begin{array}{l}\text { Acute infectious disease contributing to } \\
\text { admission }\end{array}$ & $724,531(100)$ \\
\hline Prolonged immobilization ${ }^{\text {a }}$ & $14,292(2.0)$ \\
\hline Chronic venous insufficiency or varicosis & $10,178(1.4)$ \\
\hline Recent major surgery or trauma (6-12 wk) & $27,262(3.8)$ \\
\hline Estrogen therapy & \\
\hline Chronic kidney disease, stage 3 & $(3.1)$ \\
\hline
\end{tabular}

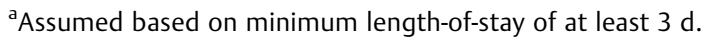

younger and healthier than seen in prior studies. ${ }^{3-6,10}$ For example, while approximately $40 \%$ of patients included in the MAGELLAN subpopulation ${ }^{3}$ were $\geq 75$ years old, only approximately $25 \%$ of patients were of that age in our study. Moreover, our study included a lower proportion of patients with heart failure $(8.4 \%)$ compared with the MAGELLAN subpopulation (approximately 37\%). ${ }^{3}$

Prior real-world studies have attempted to estimate the proportion of acute medically ill patients that might benefit from ET. ${ }^{8,10}$ A study by Rosenberg et al ${ }^{10}$ applied the International Medical Prevention Registry on Venous Thromboembolism (IMPROVE) VTE risk score to a cohort of 19,217 medically ill patients hospitalized within a tertiary health system. They found that $32 \%$ of the medically ill patients assessed would be considered high-risk for "hospital-acquired" VTE based on an IMPROVE VTE score $\geq 3$. A second study by Miao et $\mathrm{al}^{8}$ applied the Medically Ill Patient Assessment of Rivaroxaban versus Placebo in Reducing Post-Discharge Venous ThromboEmbolism Risk (MARINER) trial $^{6}$ criteria to the 2014 National Inpatient Sample

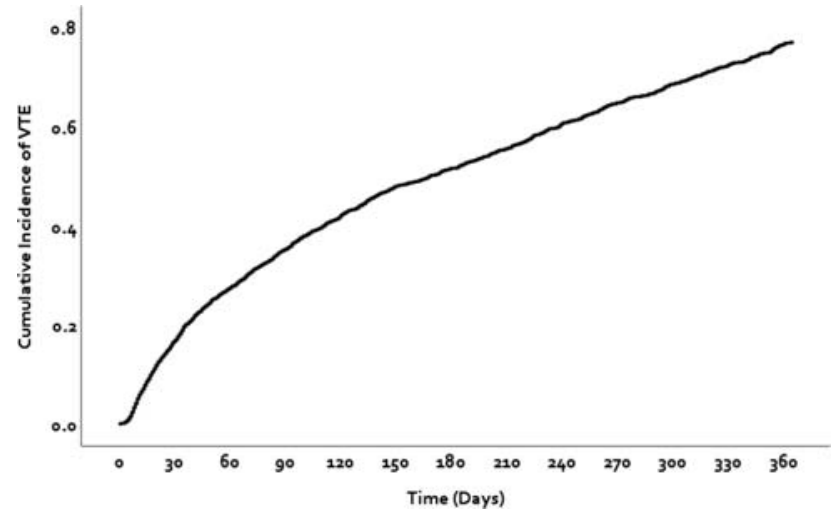

Fig. 2 Kaplan-Meier curve for time-to-first venous thromboembolism. VTE, venous thromboembolism.

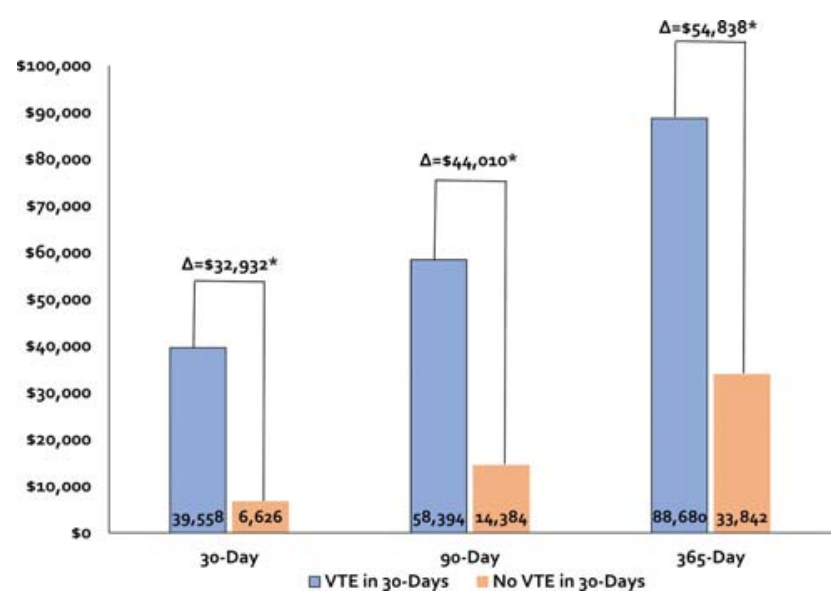

Fig. 3 Unadjusted change in 30-, 90- and 365-day costs between medically ill patients experiencing and not experiencing a venous thromboembolism in the first 30-days post-index hospitalization. $\Delta$, delta (difference in costs); VTE, venous thromboembolism. ${ }^{*} p<0.001$.

(NIS) dataset to determine the proportion of medially ill patients that might be candidates for ET. Of the $1,849,535$ hospitalizations for medical illness captured in the 2014 NIS, 407,095 hospitalizations (22.0\%) were found to be in patients at high risk for VTE (per a modified IMPROVE VTE risk score used in MARINER). These prior studies, ${ }^{8,10}$ taken together with the present, suggest that between 1 in 5 and 1 in 3 medically ill patients could be candidates for ET. Yet it is estimated that fewer than $5 \%$ of medically ill patients receive ET. ${ }^{15}$ Guidance from international/national medical societies and standards setting accrediting bodies (e.g., The Joint Commission) improved VTE risk assessment models, decision support tools integrated into electronic health records, and other quality improvement initiatives may be helpful in assuring appropriate (i.e., lower bleeding risk) at-risk medically ill patients are considered for ET.

Our study has several limitations that merit discussion. First, biases such as misclassification can negatively impact the internal validity of claims database analyses such as ours. ${ }^{14}$ Second, IBM MarketScan does not contain specific data on immobility. ${ }^{7}$ Trials have defined immobility as 
Table 2 Post-index hospitalization outcomes in subsets of patients with $>5 \%$ prevalence of an additional risk factor for venous thromboembolism

\begin{tabular}{|l|l|l|l|l|l|}
\hline Outcome & $\begin{array}{l}\text { All patients } \\
N=724,531\end{array}$ & $\begin{array}{l}\text { Morbid obesity } \\
N=39,848\end{array}$ & $\begin{array}{l}\geq 75 \mathrm{y} \\
\mathbf{N}=176,424\end{array}$ & $\begin{array}{l}\text { Heart failure } \\
\mathbf{N}=61,052\end{array}$ & $\begin{array}{l}\text { History of cancer } \\
\mathbf{N}=116,403\end{array}$ \\
\hline \multicolumn{6}{|c|}{ Venous thromboembolism, $\mathbf{n}(\%)$} \\
\hline $30 \mathrm{~d}$ & $1,532(0.2)$ & $139(0.3)$ & $141(0.1)$ & $89(0.1)$ & $242(0.2)$ \\
\hline $90 \mathrm{~d}$ & $2,727(0.4)$ & $251(0.6)$ & $310(0.2)$ & $194(0.3)$ & $472(0.4)$ \\
\hline $365 \mathrm{~d}$ & $4,487(0.6)$ & $358(0.9)$ & $798(0.5)$ & $392(0.6)$ & $856(0.7)$ \\
\hline \multicolumn{7}{|c|}{ Total costs (2018 US\$) } \\
\hline $30 \mathrm{~d}$ & $\$ 6,699 \pm 22,959$ & $\$ 7,724 \pm 26,620$ & $\$ 5,609 \pm 14,785$ & $\$ 10,071 \pm 31,543$ & $\$ 8,473 \pm 25,587$ \\
\hline $90 \mathrm{~d}$ & $\$ 14,480 \pm 40,253$ & $\$ 17,065 \pm 44,877$ & $\$ 11,850 \pm 26,162$ & $\$ 23,554 \pm 61,126$ & $\$ 18,796 \pm 45,577$ \\
\hline $365 \mathrm{~d}$ & $\$ 33,962 \pm 78,499$ & $\$ 40,345 \pm 84,370$ & $\$ 28,848 \pm 58,526$ & $\$ 53,400 \pm 118,202$ & $\$ 43,475 \pm 92,928$ \\
\hline
\end{tabular}

US\$, United States dollars.

being confined to a bed or chair for most of the day, with independent mobility only to the in-room toilet for at least 24 hours. ${ }^{4,5}$ However, outside of a prospective trial, the assessment of prolonged immobility can be difficult and/or subjective. ${ }^{16}$ In our study, we assumed an index hospital LOS $\geq 3$ days was a reasonable proxy for prolonged immobilization. ${ }^{8}$ This assumption is supported by a study by Amin and colleagues ${ }^{17}$ which identified extended hospital LOS in medically ill patients as a strong risk factor for subsequent VTE development at 6 months (with LOS of 4-6 and $\geq 7$ days associated with a 41 and $221 \%$ increased risk of VTE compared with a LOS of 1-3 days). However, it is possible our use of LOS as a proxy for prolonged immobility resulted in an overestimation of patient risk. Third, we classified a medically ill patient's reason for hospitalization based upon their primary billing code for that hospitalization (the sum of the proportion of reasons for hospitalization total to $100 \%$ ) making direct comparison of our demographic data to that of prior randomized trials ${ }^{3-6}$ difficult. It is likely that many patients in our study had more than one diagnosis (i.e., heart failure, respiratory insufficiency, ischemic stroke, infection, or inflammatory disease) and/or risk factor qualifying them for ET. Fourth, although we adjusted for demographics, primary reason for the hospitalization and 12 key comorbidities/risk factors for VTE in our regression analyses estimating differences in post-index hospitalization costs between patients experiencing and not experiencing an early VTE, residual confounding cannot be ruled out. Moreover, our analysis does not provide an estimate of the proportion of the difference in costs that are directly attributable to the management of VTE or its complications. Finally, we performed this analysis in the IBM MarketScan CCAE and Medicare supplemental databases. While this combined dataset covers many lives spanning all age groups, ${ }^{7}$ it is skewed toward a younger and healthier medically ill population (particularly compared with randomized trials) which may have resulted in an underestimation of the proportion of patients eligible for ET in the real world. The use of MarketScan data also makes our findings less generalizable to non-U.S. patients.

\section{Conclusion}

Our study found that $26 \%$ of the medically ill patients evaluated met the MAGELLAN subpopulation criteria for use of rivaroxaban for ET. Early post-discharge VTE occurrence in these patients was associated with substantially increased total downstream health care costs. Steps to reduce patients' risk of developing hospital acquired VTE after discharge should be implemented using ET with approved direct oral anticoagulants.

Funding

This study was funded by Janssen Scientific Affairs LLC, Titusville, Florida.

\section{Conflict of Interest}

C.I.C. reports grants from Janssen Scientific Affairs LLC, during the conduct of the study; grants, personal fees, and non-financial support from Janssen Scientific Affairs LLC, grants, personal fees and nonfinancial support from Bayer AG, grants and personal fees from Portola Pharmaceuticals, outside the submitted work. G.P. reports personal fees and nonfinancial support from Janssen Scientific Affairs LLC, during the conduct of the study; grants and personal fees from Janssen Scientific Affairs LLC, grants and personal fees from Portola Pharmaceuticals, grants and personal fees from Bristol-Myers Squibb/Pfizer, grants and personal fees from BTG/EKOS, grants and personal fees from Daiichi-Sankyo, grants and personal fees from Amgen, grants and personal fees from Boston Scientific, outside the submitted work. A.C.S. reports personal fees and nonfinancial support from Janssen Scientific Affairs LLC, during the conduct of the study; grants and personal fees from Janssen Pharmaceuticals, grants and personal fees from Portola Pharmaceuticals, grants and personal fees from Bristol-Myers Squibb, grants and personal fees from Boehringer Ingelheim Pharmaceuticals, grants and personal fees from Bayer AG, grants and personal fees from the ATLAS Group, outside the submitted work. V.A. reports other from Janssen Scientific Affairs LLC, outside the submitted work. 


\section{References}

1 Cohen AT, Alikhan R, Arcelus JI, et al. Assessment of venous thromboembolism risk and the benefits of thromboprophylaxis in medical patients. Thromb Haemost 2005;94(04): 750-759

2 Samama MM. An epidemiologic study of risk factors for deep vein thrombosis in medical outpatients: the Sirius study. Arch Intern Med 2000;160(22):3415-3420

3 Spyropoulos AC, Lipardi C, Xu J, et al. Improved benefit risk profile of rivaroxaban in a subpopulation of the MAGELLAN study. Clin Appl Thromb Hemost 2019;25:1076029619886022

4 Cohen AT, Spiro TE, Büller HR; MAGELLAN Investigators, et al. Rivaroxaban for thromboprophylaxis in acutely ill medical patients. N Engl J Med 2013;368(06):513-523

5 Cohen AT, Harrington RA, Goldhaber SZAPEX Investigators, et al. APEX Investigators. Extended thromboprophylaxis with betrixaban in acutely ill medical patients. N Engl J Med 2016;375(06): 534-544

6 Spyropoulos AC, Ageno W, Albers GWMARINER Investigators, et al. Rivaroxaban for thromboprophylaxis after hospitalization for medical illness. N Engl J Med 2018;379(12):1118-1127

7 Hansen L. The Truven Health MarketScan Databases for Life Sciences Researchers. Available at: https://truvenhealth.com/ Portals/0/Assets/2017-MarketScan-Databases-Life-SciencesResearchers-WP.pdf. Accessed March 1, 2019

8 Miao B, Chalupadi B, Clark B, et al. Proportion of US hospitalized medically ill patients who may qualify for extended thromboprophylaxis. Clin Appl Thromb Hemost 2019;25:1076029619850897

9 White RH, Garcia M, Sadeghi B, et al. Evaluation of the predictive value of ICD-9-CM coded administrative data for venous thromboembolism in the United States. Thromb Res 2010;126(01):61-67
10 Rosenberg D, Eichorn A, Alarcon M, McCullagh L, McGinn T, Spyropoulos AC. External validation of the risk assessment model of the International Medical Prevention Registry on Venous Thromboembolism (IMPROVE) for medical patients in a tertiary health system. J Am Heart Assoc 2014;3(06):e001152

11 US Bureau of Labor Statistics Consumer price index Available at: https://www.bls.gov/cpi/. Accessed April 28, 2020

12 Benchimol EI, Smeeth L, Guttmann ARECORD Working Committee, et al; The REporting of studies conducted using observational routinely-collected health data (RECORD) statement. PLoS Med 2015;12(10):e1001885

13 XARELTO ${ }^{\circledR}$ (rivaroxaban) tablets, for oral use Prescribing information Janssen Pharmaceuticals, Inc., Titusville, NJ, March 2020 Available at: http://www.janssenlabels.com/package-insert/product-monograph/prescribing-information/XARELTO-pi. pdf. Accessed April 28, 2020

14 Gandhi SK, Salmon W, Kong SX, Zhao SZ. Administrative databases and outcomes assessment: an overview of issues and potential utility. J Manag Care Spec Pharm 1999;5(03):215-222

15 Mahan CE, Burnett AE, Fletcher ML, Spyropoulos AC. Extended thromboprophylaxis in the acutely ill medical patient after hospitalization-a paradigm shift in post-discharge thromboprophylaxis. Hosp Pract (1995) 2018;46(01):5-15

16 Hull RD. Relevance of immobility and importance of risk assessment management for medically ill patients. Clin Appl Thromb Hemost 2013;19(03):268-276

17 Amin A, Neuman WR, Lingohr-Smith M, Menges B, Lin J. Influence of the duration of hospital length of stay on frequency of prophylaxis and risk for venous thromboembolism among patients hospitalized for acute medical illnesses in the USA. Drugs Context 2019;8:212568 